Historic, Archive Document

Do not assume content reflects current scientific knowledge, policies, or practices. 

Terms of payment. Half (1-2) cash with order. Balance Junc 1st. Boxing and burlap at actual cost.

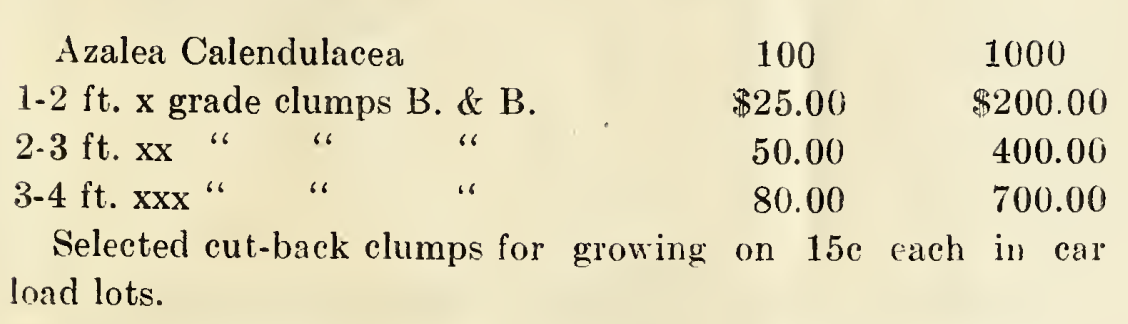

Azalea Viscosa and Canescens same price as Calendulacea.

\section{Kalmia latifolia}

1-2 ft. Select stocky clumps

$2-3 \mathrm{ft} . \mathrm{xx}$

"،
"،
"

25.00

45.00

75.00

$3-4 \mathrm{ft} . \mathrm{xxx}$

125.00

$4-5$ ft. $\mathrm{xxxx}$

200.00

Leusothoe Catesbaei

1.2 ft. Select clumps B. \& B.

$2-3 \mathrm{ft} . \mathrm{xx} \quad$ " "

$3-4 \mathrm{xxx}$ " "

Nice cut back clumps for growing on $20 \mathrm{c}$ each.

Rhododendron carolinianum

1-2 ft. Selecterl Clumps B. \& B.

2-3 ft. $\mathrm{xx}$

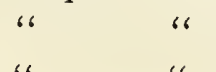

$3-4 \mathrm{ft} . \mathrm{xxx}$

" "

$4-5 \mathrm{ft} . \mathrm{xxx}$

" "

$5-6 \mathrm{ft}$. $\mathrm{xxx}$

" $"$ "

30.00

50.00

80.00

Select cut-back clumps 22c each.

\section{Catawbiense}

$1-2 \mathrm{ft}$. Select stocky clumps

2-3 ft. $x x$

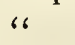

$3-4$ ft. $x x x$

"

$4-5 \mathrm{ft} . \mathrm{xxx}$

5-6 ft. $\mathrm{xxx}$

6

"

Cut-back clumps 22c each.

\section{Maximum}

$1 \cdot 2 \mathrm{ft}$. select clumps

$2-3$ ft. $x \mathbf{x}$

$3-4$ ft. $\mathrm{xxx}$

$4.5 \mathrm{ft} . \mathrm{xxx}$

".

“

$56 \mathrm{ft} . \mathrm{xxx}$

Cut-back clumps 15c each.

200.00

400.00

700.00

1000.00

1750.00

225.00 750.00
70.00

650.00

90.00

160.00

200.00

250.00

850.00

1400.00

1800.00

2200.00

500.910

$95 \mathrm{C} .00$

1600.00

2000.00

2750.00

225.00

300.00

\section{TAR HEEL NURSERY \\ 3 \\ R. B. Dula \\ COLLECTOR AND "' NURSERYMAN \\ NEWLAND, N. C.} $375.00 \times 50$

\section{LINING OUT STOCK}

Rhododendron Maximum

3-6 in. Strong seedlings

$6-12$ in. " " "
12.18 in "

Kalmia latifolia

3.6 in. strong seedlings

$6-12$ in. " "

$12-18$ in. " "

Leucothoe catesbaei

$3-6$ in. strong seedlings

$6-12$ in. " “ "

$12-18$ in.

0.00

40.00

Abies fraseri

$3 \cdot 6$ in. strong seedlings

$6 \cdot 12$ in. " " " 12.18 in "

20.00

60.00

120.00

Ilex opaca

$3-6$ in. strong seedlings $\quad 30.00$

6-I2 in. " " $\quad 6000$

1218 in. " " $\quad 120.00$

Tsuga car oliniana

$3-6$ in. strong seedlings

$6-12$ in. " "

30.00

$12 \cdot 18$ in." "

Leiophyllum buxifolium (sand myrtle)

$3 \cdot 6$ in. nice clumps

6-12 in. strong clumps

1218 in. " "

120.00

200.00

250.00

I ris cristata

strong clumps

40.00

E jiguea repens (trailing arbutus)

Strong cumps

40.00

Galax aphylla

Strong clumps

\section{HARDY NATIVE FERNS}

40.00

Adiantum pedatum

Aspidium acrostichoides (dagger fern)

Aspidium marginale (shield fern)

Aspidium spinulosum

1000

$\$ 40.00$

40.00

40.00

40.00

Dicksoniana punctilobula

(Hay scented fern)

2000

Osmunda cinnamomea

Cinnamon fern

60.00

Osmunde claytoniana

(Interrupted fern)

Polypodium vulgare

30.00

Ferns are cut in nice, square clumps.

Prices gladly quoted on anything not listed. 
